\title{
Simulation of AGN feedback and its impact on galaxies
}

\author{
Martin A. Bourne \\ Institute of Astronomy and Kavli Institute for Cosmology, University of Cambridge, \\ Madingley Road, Cambridge, CB3 0HA, UK \\ email: mabourne@ast.cam.ac.uk
}

\begin{abstract}
Feedback released during the growth of supermassive black holes is expected to play a key role in shaping black hole-host galaxy co-evolution. Powerful, accretion disc driven winds have been invoked to explain both observed scaling relations (e.g., $M-\sigma$ ) and large-scale outflows with mass outflow rates of $\sim 100-1000 \mathrm{M}_{\odot} \mathrm{yr}^{-1}$ and momentum rates of up to $\sim 30$ $\mathrm{L}_{\mathrm{AGN}} / \mathrm{c}$. Critically, how these winds couple to the host galaxy depends on if they are momentum or energy conserving. I outline observational signatures that could distinguish between these regimes and discuss their roles in establishing galaxy properties. Furthermore, I discuss highresolution simulations exploring feedback in a multi-phase medium, highlighting how structural properties of galaxies can impact feedback efficiency. Finally, feedback, in the form of collimated jets, is expected to regulate cooling in galaxy clusters. I discuss new simulations of jet feedback using the moving-mesh code AREPO and outline the scope of our new study.
\end{abstract}

Keywords. galaxies: active, galaxies: ISM, (galaxies:) quasars: general, galaxies: clusters: general, (galaxies:) cooling flows, galaxies: evolution, galaxies: jets, methods: numerical

\section{Introduction}

It is widely accepted that at the centres of most massive galaxies resides a supermassive black hole (SMBH). Active galactic nuclei (AGN) represent phases of accretion onto the $\mathrm{SMBH}$ that results in the release of vast amounts of energy or feedback. This feedback is expected to play an important role in shaping the host galaxy and has become a key ingredient in many galaxy formation models and simulations (Di Matteo et al., 2005, Bower et al., 2006, Croton et al., 2006, Sijacki et al., 2015). Scaling relations between the properties of the central SMBH and the host galaxy, such as $M-\sigma$ (Kormendy \& Ho, 2013), are usually interpreted as evidence for feedback mediated co-evolution. Comparing the energy released growing a SMBH to it's $M-\sigma$ mass, $E_{\mathrm{BH}} \sim \eta M_{\mathrm{BH}} c^{2}$, with the binding energy of the gas within a bulge $E_{\mathrm{b}} \sim f_{\mathrm{g}} M_{\mathrm{b}} \sigma^{2} \sim 200 f_{\mathrm{g}} M_{\mathrm{BH}} \sigma^{2}$, one finds a ratio of $\sim 10^{3}-10^{4}$. This suggests firstly that more than enough energy is released to have a serious impact on the gas within a galaxy and secondly that the energy released must couple very weakly (see, King \& Pounds, 2015, for a review).

Broadly, AGN feedback can be split into two categories (see e.g., Fabian, 2012); quasar mode feedback, which represents phases of rapid SMBH growth, at high Eddington ratios and radio mode feedback, which is expected during more sedate episodes of accretion with lower Eddington ratios. Feedback during the quasar mode is expected to take the form of winds and outflows which push gas out of the galaxy, quench star formation and facilitate the production of the $M-\sigma$ relation. On the other hand, the radio mode is expected to proceed through the production of highly collimated jets, which act to regulate heating and cooling within the hot haloes of galaxies, for example in galaxy clusters, preventing gas cooling onto the galaxy and hence inhibiting further star formation. 


\section{AGN winds and outflows}

Large scale outflows, likely driven by small scale AGN winds, have been observed in a number of objects (e.g.,Rupke \& Veilleux, 2011, Sturm et al., 2011, Cicone et al., 2014, Cicone et al., 2015, Feruglio et al., 2015, Tombesi et al., 2015) out to kpc scales and across a range of redshifts $(z \gtrsim 6)$. They are seen in ionised, neutral atomic and molecular gas with large mass outflow rates of $\dot{M}_{\text {out }} \sim 100-1000 \mathrm{M}_{\odot} \mathrm{yr}^{-1}$, high velocities $v_{\text {out }} \sim 1000$ $\mathrm{km} \mathrm{s}^{-1}$, high energy content $\dot{E}_{\text {out }} \sim 0.05 L_{\mathrm{AGN}}$ and large momentum boosts of up to $\sim 30 \times L_{\mathrm{AGN}} / c$ (Faucher-Giguère et al., 2012, King \& Pounds, 2015). The kinetic energy content of these winds therefore requires that a few percent of the total AGN luminosity couples to the interstellar medium (ISM) to drive these outflows. Yet the $M-\sigma$ relation, if driven by AGN feedback, suggests much weaker coupling.

Fast accretion disc winds, such as ultra-fast outflows (UFOs) observed in blue shifted X-ray absorption features (Tombesi et al., 2010, Tombesi et al., 2010), have provided a particularly successful feedback channel to explain both the $M-\sigma$ relation and large-scale outflows. The UFOs, which have fairly simple properties $-v_{\mathrm{w}} \sim 0.1 \mathrm{c}, \dot{E}_{\mathrm{w}} \sim 0.05 L_{\mathrm{Edd}}$ and $\dot{p}_{\mathrm{w}} \sim L_{\mathrm{Edd}} / c$ (King \& Pounds, 2015), collide with and shock against the ISM, forming a reverse wind shock and forward ISM shock. The wind shock will reach temperatures of $T_{\mathrm{ws}} \sim 10^{10} \mathrm{~K}$ and subsequent evolution of the outflow depends on whether or not the wind shock retains the thermalised kinetic energy. If the wind shock can cool efficiently, then it is in the momentum conserving regime and only the pre-shock ram pressure drives the large-scale outflow. King $(2003,2005)$ has shown that a momentum conserving outflow results in a limiting SMBH mass similar to the $M-\sigma$ relation. However, if the wind shock is unable to cool, i.e., it is energy conserving, then the thermal pressure of the shocked wind bubble drives the outflow. This regime could potentially have a much greater impact on the host galaxy ISM and can explain both the energy content and momentum boosts observed in large-scale outflows (Faucher-Giguère \& Quataert, 2012, Zubovas \& King, 2012).

\subsection{Inverse-Compton cooling of the wind shock}

The dominant cooling mechanism of the wind shock would likely be inverse-Compton (IC) cooling of the wind shock electrons by the AGN radiation field photons (King, 2003). Assuming a single temperature plasma for the wind shock, IC cooling is effective within the central $\sim 500 \mathrm{pc}$ of a galaxy (King, 2003) resulting in an outflow that is momentum driven within this radius and energy driven beyond it (King, 2005). In this model, the $M-\sigma$ relation can be established at small radii while at larger radii powerful outflows can be produced. Given that IC cooling only acts on the electrons, there needs to be efficient coupling between the protons, which carry most of the energy, and the electrons in the wind shock. However, Faucher-Giguère \& Quataert (2012) have shown that the low-density, high-temperature conditions mean that energy exchange between protons and electrons through Coulomb collisions is inefficient and hence IC cooling is ineffective. We, therefore, proposed an observational test of cooling.

In Bourne \& Nayakshin (2013), we calculated the IC signature of the AGN photons up-scattered by the hot wind electrons. Assuming that all of the wind kinetic energy is radiated, the components have luminosities of $L_{\mathrm{IC}}=(1 / 2) \dot{M}_{\mathrm{w}} v_{\mathrm{w}}^{2}$ and so higher wind velocities produce higher luminosity components. Further, increased wind velocities result in higher shock temperatures and hence higher energy photons can be produced. This is illustrated in Fig. 4 of Bourne \& Nayakshin (2013); as the wind velocity increases the IC component moves from being predominantly in softer X-rays with low luminosity to being in harder X-rays with high luminosity. For velocities of $v_{\mathrm{w}}=0.1 \mathrm{c}$ and above the component becomes comparable to a typical AGN spectrum and we expect such a component to be readily observable if it exists. However, the only observed component 
we are aware of is in NGC 4051 (Pounds \& Vaughan, 2011), which is at a radius of less than 1 pc. The lack of observed components on larger scales, beyond the central few parsecs, and the theoretical modelling of Faucher-Giguère \& Quataert (2012), points towards outflows essentially always being energy driven. Therefore we pose the question, how can SMBHs grow so massive?

\subsection{AGN feedback in a multi-phase medium}

In an attempt to answer this question, we performed hydrodynamical simulations, presented in Bourne et al. (2014). We compared the evolution of a hot gas bubble, mimicking the energy conserving hot wind shock, in a homogeneous versus inhomogeneous medium. The homogeneous scenario behaves as expected, with the hot bubble expanding and sweeping up all material in its path. On the other hand, in a turbulent and inhomogeneous medium, the hot bubble escapes via low-density channels, while high-density gas is affected far less. High-density clumps can be compressed (potentially triggering star formation), ablated or even completely destroyed depending on their location and physical properties.

Interestingly, while in the homogeneous case mass and energy flows are dominated by outflowing material, this is not the case for a turbulent and inhomogeneous medium. In the latter, we find that the mass and energy flows are able to decouple, with the energy flow being dominated by outflowing material and the mass flow tending to consist more of in-flowing gas. This provides a situation in which, despite feedback occurring, most of the energy can escape the system relatively freely while gas clumps can continue to flow inwards, potentially providing fuel for SMBH growth. Similar to previous work (e.g., Wagner et al., 2013), we find that it is the ram pressure of the outflow that acts on the high-density clumps and thus it is the clump column density that determines whether or not a clump can be swept outwards. Assuming that the column density of dense clumps is limited by star formation, Nayakshin (2014) has shown that the $M-\sigma$ relation can be recovered.

\section{Jet mode feedback}

As well as the quasar mode of feedback discussed in the previous section, a second major mode of AGN feedback exists as the jet mode. To investigate this regime we have implemented a jet feedback routine into the moving mesh code AREPO (Springel, 2010), making use of the super-Lagrangian refinement scheme of Curtis \& Sijacki (2015). This scheme allows improved resolution close to the SMBH, which in turn gives the ability to better capture gas flows close to the SMBH and in the case of this work, inject a jet on small scales. Similar to other works (e.g., Omma et al., 2004, Cattaneo \& Teyssier, 2007), we inject mass, momentum and energy into a cylinder centred on the SMBH in order to produce the jet. We have compared various jet injection techniques including pure momentum driven jets as well as purely kinetic jets and jets with a momentum and thermal component, finding marked differences in their evolution and content of different energy components. Further, we have tested precessing kinetic jets and compared these to those mentioned previously. We have been able to study the evolution of the jets, the inflation of cocoons and their subsequent interaction with the intracluster medium (ICM), including measurements of jet-induced turbulence. A detailed account of this research will be presented in future publications that are currently in preparation.

\section{Summary}

SMBH scaling relations suggest that coupling of AGN feedback to the ISM is weak and potentially it is only the momentum that is important in establishing scaling relations, 
although the plethora of physical processes involved means this is still rather uncertain. Yet, the powerful outflows observed on $\mathrm{kpc}$ scales require that approximately a few percent of the AGN energy couples to the host ISM. If IC cooling of the wind shock occurs, we predict that it should produce an X-ray feature, which has not been observed at large radii. This lack of an observation could hint at inefficient IC cooling, potentially due to weak electron-proton coupling (Faucher-Giguère \& Quataert, 2012). Modelling structure in the ISM in simulations makes it more resilient to AGN feedback, even in the energy driven regime. It is the ram pressure of the outflow that is communicated to the bulk of the gas and this may, therefore, negate the need for IC cooling of the wind shock in order to explain both the $M-\sigma$ relation and powerful large scale outflows.

Finally, we have implemented a jet feedback scheme into AREPO in combination with a super-Lagrangian refinement technique that allows the jet to be injected on small scales. We have compared various jet implementation schemes to investigate their impact on both jet and halo properties. Preliminarily it appears that the jet is unable to drive significant bulk turbulence within the ICM, which is consistent with Hitomi observations of the Perseus Cluster (Hitomi Collaboration et al., 2016).

\section{Acknowledgements}

I would like to acknowledge support by the ERC starting grant 638707 "BHs and their host galaxies: co-evolution across cosmic time."

\section{References}

Bourne, M. A., Nayakshin, S., \& Hobbs, A. 2014, MNRAS, 441, 3055

Bourne, M. A. \& Nayakshin, S. 2013, MNRAS, 436, 2346

Bower, R. G., Benson, A. J., Malbon, R., et al. 2006, MNRAS, 370, 645

Cattaneo, A. \& Teyssier, R. 2007, MNRAS, 376, 1547

Cicone, C., Maiolino, R., Sturm, E., et al. 2014, A\&AA, 562, A21

Cicone, C., Maiolino, R., Gallerani, S., et al. 2015, A\& $A$, 574, A14

Croton, D. J., Springel, V., White, S. D. M., et al. 2006, MNRAS, 365, 11

Curtis, M. \& Sijacki, D. 2015, MNRAS, 454, 3445

Di Matteo, T., Springel, V., \& Hernquist, L. 2005, Nature, 433, 604

Fabian, A. C. 2012, ARAA, 50, 455

Faucher-Giguère, C.-A., Quataert, E., \& Murray, N. 2012, MNRAS, 420, 1347

Faucher-Giguère, C.-A. \& Quataert, E. 2012, MNRAS, 425, 605

Feruglio, C., Fiore, F., Carniani, S., et al. 2015, A\&A, 583, A99

Hitomi Collaboration, Aharonian, F., Akamatsu, H., et al. 2016, Nature, 535, 117

King, A. 2003, ApJ, 596, L27

King, A. 2005, $A p J, 635$, L121

King, A. \& Pounds, K. 2015, ARAA, 53, 115

Kormendy, J. \& Ho, L. C. 2013, ARAA, 51, 511

Nayakshin, S. 2014, MNRAS, 437, 2404

Omma, H., Binney, J., Bryan, G., \& Slyz, A. 2004, MNRAS, 348, 1105

Pounds, K. A. \& Vaughan, S. 2011, MNRAS, 413, 1251

Rupke, D. S. N. \& Veilleux, S. 2011, ApJ, 729, L27

Sijacki, D., Vogelsberger, M., Genel, S., et al. 2015, MNRAS, 452, 575

Springel, V. 2010, MNRAS, 401, 791

Sturm, E., González-Alfonso, E., Veilleux, S., et al. 2011, ApJ, 733, L16

Tombesi, F., Cappi, M., Reeves, J. N., et al. 2010, A\&A, 521, A57

Tombesi, F., Sambruna, R. M., Reeves, J. N., et al. 2010, ApJ, 719, 700

Tombesi, F., Meléndez, M., Veilleux, S., et al. 2015, Nature, 519, 436

Wagner, A. Y., Umemura, M., \& Bicknell, G. V. 2013, ApJ, 763, L18

Zubovas, K. \& King, A. 2012, ApJ, 745, L34 